\title{
Modelación Matemática para la Dinámica de Mecanismos Hidráulicos Tipo Tijera
}

\section{Mathematical Modeling for the Dynamics of Scissors Mechanisms}

Sócrates Miguel Aquino Arroba. ${ }^{1}$, Luis Patricio Tierra Pérez. ${ }^{2}$, Edwin Rodolfo Pozo Safla.

${ }^{3}$ \& Eduardo Santiago Cazar Rivera. ${ }^{4}$

Recibido: 28-04-2019 / Revisado: 04-05-2019 /Aceptado: 28-06-2019/ Publicado: 15-07-2019

\begin{abstract}
.
DOI: https://doi.org/10.33262/cienciadigital.v3i3.1.677

The objective of this document was the kinematic and dynamic analysis of the mechanism of a scissor-type mechanical cat focused on design verification, identifying the different types of mechanism that provide a solution to the problem of lifting a load of 0.5 tons corresponding to a Renault Clio car. The material from which it is constructed was also determined through bibliographic references for the evaluation with finite element software. The parameters and dimensions were obtained through field research, where the mechanism measurements were taken. The results obtained allowed establishing that the mechanism meets a safety factor between 1 and 1.7 where it is verified that the mechanism is optimized to the maximum.
\end{abstract}

Keywords: Analysis, Dynamics, Elements, Finites, Lift, Mechanic.

\section{Resumen.}

La presente investigación tiene por objeto el análisis cinemático y dinámico del mecanismo de una gata mecánica tipo tijera enfocado a la verificación del diseño, identificando los distintos tipos de mecanismo que den solución al problema del levantamiento de una carga de 0,5 toneladas correspondiente a un automóvil Renault Clio. Se determinó también el material del que está construido mediante referencias

1 Escuela Superior Politécnica de Chimborazo, Facultad de Mecánica. Riobamba, Ecuador, miguel. aquino@espoch.edu.ec.

2 Escuela Superior Politécnica de Chimborazo, Facultad de Recursos Naturales, Macas, Ecuador, patricio.tierra@espoch.edu.ec.

3 Escuela Superior Politécnica de Chimborazo, Facultad de Mecánica. Riobamba, Ecuador, edwin.pozo@espoch.edu.ec.

4 Escuela Superior Politécnica de Chimborazo, Facultad de Recursos Naturales, Macas, Ecuador, eduardo.cazar@espoch.edu.ec. 
bibliográficas para la evaluación con software de elementos finitos. Los parámetros y dimensiones fueron obtenidos mediante investigación de campo, en donde se tomaron las medidas del mecanismo. Los resultados obtenidos permitieron establecer que el mecanismo se encuentra con un factor de seguridad entre 1 y 1,7 en donde se verifica que el mecanismo está optimizado al máximo.

Palabras Claves: Análisis, Dinámica, Elementos, Finitos, Gata, Mecánica.

\section{Introducción.}

El gato mecánico es una herramienta empleada para la elevación de cargas mediante el accionamiento manual de una manivela o una palanca (Uicker, 2016), se diferencia de dos tipos según su principio de funcionamiento: Gato mecánico y Gato hidráulico (Cuenca, 2000). El gato tipo tijera es del tipo mecánico suele ser el más común en los coches de carretera y funciona con un mecanismo de tornillo su popularidad se debe a su capacidad de generar una gran ventaja mecánica (Ordoñez V, et al., 2018), es decir, una gran ampliación de fuerza a partir de un brazo manual (Industrial, 2011).

En este trabajo se utiliza cinemática inversa, ya que determinan los valores que deben adoptar las coordenadas articulares del mecanismo $\left[q_{1}, q_{2}, q_{3}\right]$ para que su extremo se posicione y oriente según una determinada localización espacial (Oliva C et al., 2019).

Hipótesis. - En la presente investigación se pretende hacer una análisis cinético - dinámico para ver la resistencia de un mecanismo de gata mecánica tipo tijera, bajo determinadas condiciones, verificando su resistencia y determinando los parámetros requeridos para colocarlo en determinada posición.

\section{Materiales y Métodos.}

A lo largo de la historia el hombre ha creado diversos mecanismos ante la necesidad de satisfacer sus necesidades. Los avances de la tecnología permiten que hoy por hoy el diseño pueda ser probado validado incluso antes de ser construido, en este análisis se aplican las técnicas de la materia de Dinámica de Máquinas (Cuenca M, 2000) para evidenciar el proceso de análisis que se pueden realizar a los mecanismos para garantizar su funcionalidad. Demostrando que son técnicas de gran valor para la ciencia y por lo tanto para la humanidad.

Se requiere un mecanismo que permita levantar objetos de grandes masas, como un automóvil de media tonelada, con la fuerza que puede proporcionar una persona sin realizar esfuerzos excesivos que puedan afectar su salud física (Tierra P et al, 2018).

Se define la aplicación de una gata mecánica tipo tijera analizando mecanismo eslabonado simple. Las condiciones de funcionamiento se establecen de una gata mecánica obtenida del automóvil Renault Clio 2005, que tiene un peso de 0.5 toneladas, que es equivalente a 500 
kilogramos. Por tal motivo la gata mecánica deberá asegurar que el peso a soportar será el mismo del automóvil.

Según las consideraciones de fabricación de una gata mecánica, se define que el material a utilizar será el acero SAE 1035, estos aceros son seleccionados en usos donde se necesitan propiedades mecánicas más elevadas y frecuentemente llevan tratamiento térmico de endurecimiento (Torres E, 2004), (Forn A, 2002).

Para el análisis cinemático y dinámico de la gata mecánica tipo tijera se obtendrán las dimensiones generales como se muestra en la figura 1:

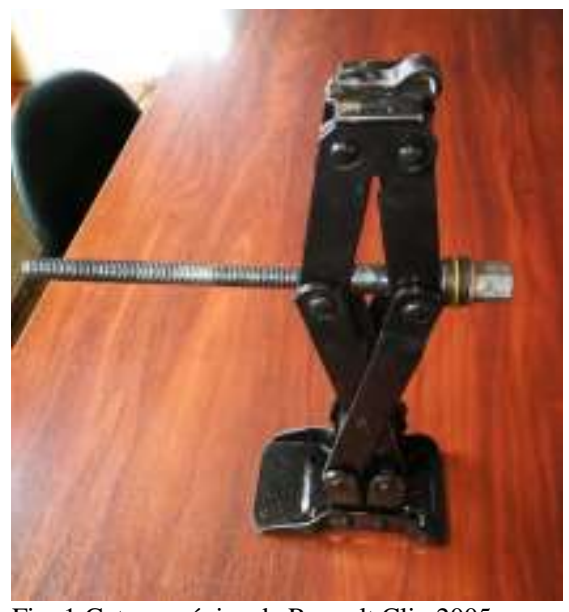

Fig. 1 Gata mecánica de Renault Clio 2005

Luego de haber realizado las mediciones principales del mecanismo, se obtiene el siguiente esquema para el análisis cinemático y dinámico:

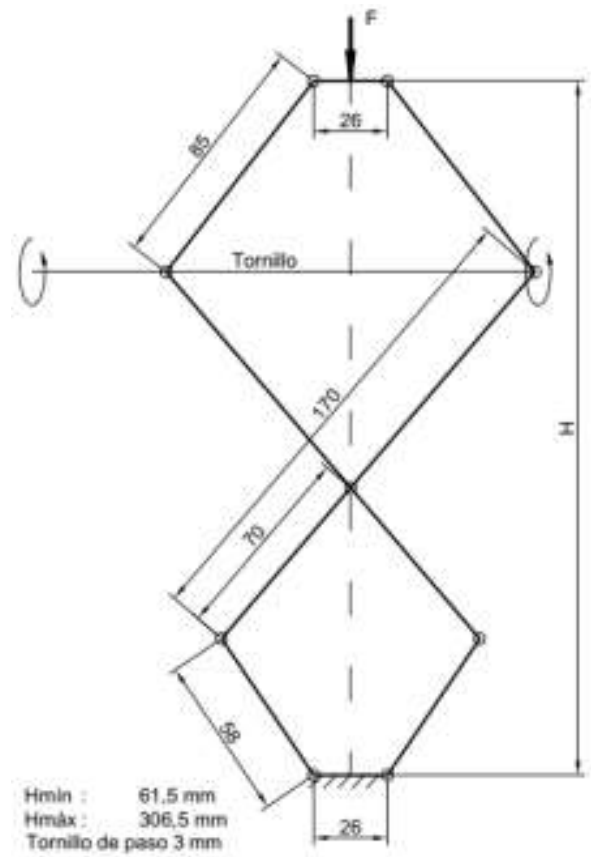

Fig. 2 Esquema general de la gata mecánica 
Para el estudio cinemático, se tomará en cuenta la mitad del mecanismo, ya que es simétrico:

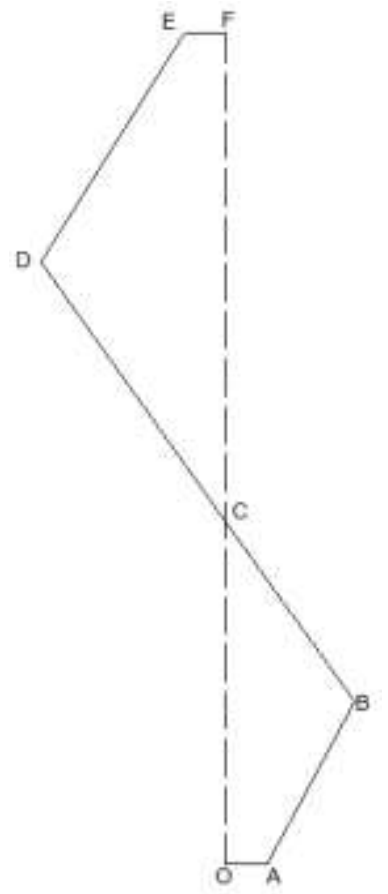

Fig. 3 Mecanismo para análisis cinemático

Para determinar las ecuaciones de movimiento (Snider A, 2001), se realiza una suma vectorial, de la siguiente manera:

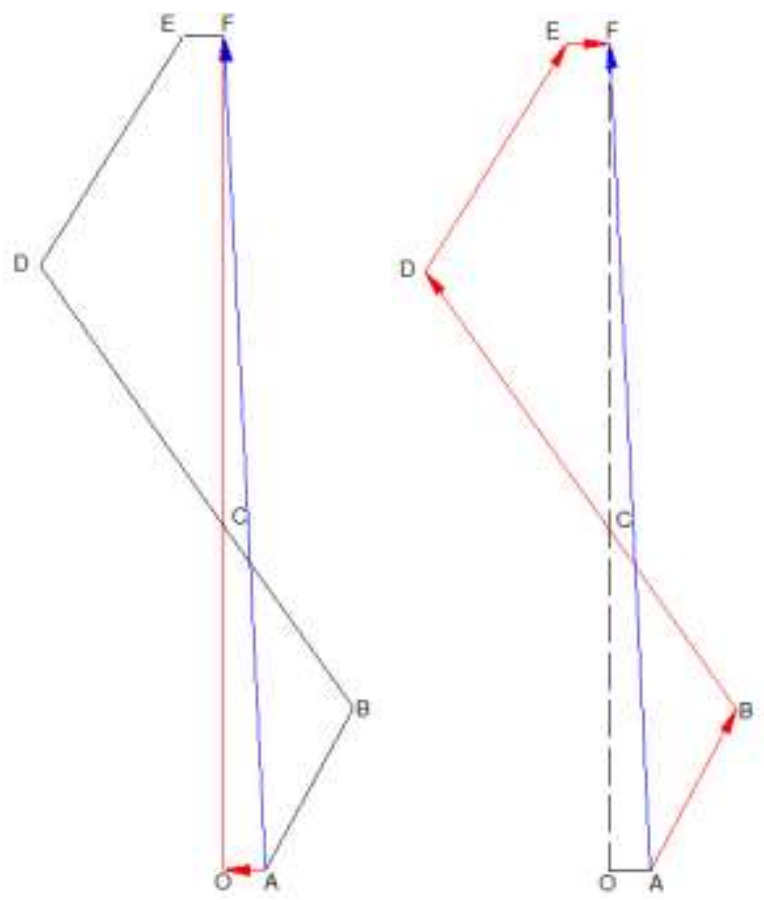

Fig. 3 Mecanismo para análisis cinemático 
De la figura 2 y figura 3 se obtuvieron las siguientes ecuaciones de posición, velocidad y aceleración (Felker D, \& Hill A, 2005), las funciones son:

Posición:

$$
\left\{\begin{array}{c}
f_{1}=A B \cdot C q_{1}+B D \cdot C q_{2}+D E \cdot C q_{3}+E F+A O \\
f_{2}=A B \cdot S q_{1}+B D \cdot S q_{2}+D E \cdot S q_{3}-q_{4}
\end{array}\right.
$$

Velocidad:

$$
\left\{\begin{array}{c}
f_{1}^{\prime}=-A B \cdot S q_{1} \cdot \dot{q}_{1}-B D \cdot S q_{2} \cdot \dot{q}_{2}-D E \cdot S q_{3} \cdot \dot{q}_{3} \\
f_{2}^{\prime}=A B \cdot C q_{1} \cdot \dot{q}_{1}+B D \cdot C q_{2} \cdot \dot{q}_{2}+D E \cdot C q_{3} \cdot \dot{q}_{3}-\dot{q}_{4}
\end{array}\right.
$$

Aceleración:

$$
\left\{\begin{array}{c}
f_{1}^{\prime \prime}=-A B \cdot C q_{1} \cdot \dot{q}_{1}^{2}-A B \cdot S q_{1} \cdot \ddot{q}_{1}-B D \cdot C q_{2} \cdot \dot{q}_{2}{ }^{2}- \\
\text { BD.S } q_{2} \cdot \ddot{q}_{2}-D E \cdot C q_{3} \cdot \dot{q}_{3}{ }^{2}-D E \cdot S q_{3} \cdot \ddot{q}_{3} \\
f_{2}^{\prime \prime}=-A B \cdot S q_{1} \cdot \dot{q}_{1}{ }^{2}+A B \cdot C q_{1} \cdot \ddot{q}_{1}-B D \cdot S q_{2} \cdot \dot{q}_{2}{ }^{2}+ \\
B D \cdot C q_{2} \cdot \ddot{q}_{2}-D E \cdot S q_{3} \cdot \dot{q}_{3}{ }^{2}+D E \cdot C q_{3} \cdot \ddot{q}_{3}-\ddot{q}_{4}
\end{array}\right.
$$

Para solucionar el sistema de ecuaciones por el Método de Newton-Euler se requiere de una ecuación adicional, la que se halla por triángulos semejantes según la figura 4:

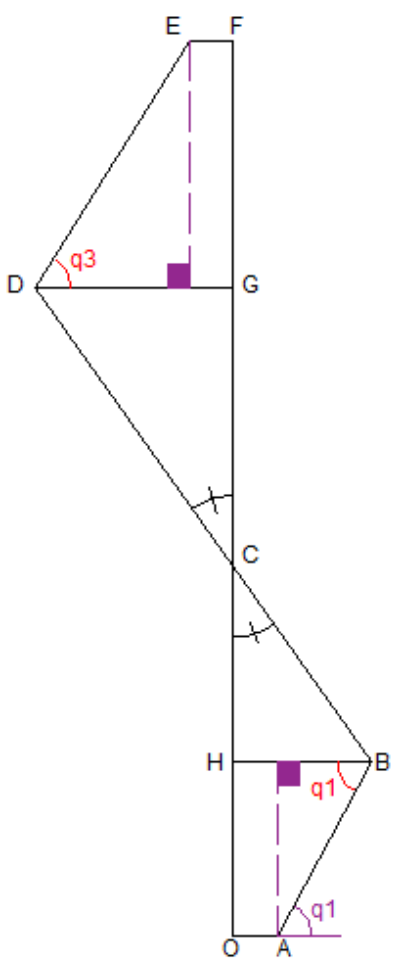

Fig. 4 Semejanza de triángulos 
Las ecuaciones de posición, velocidad y aceleración según la figura 4 son:

$$
\begin{gathered}
f_{3}=C D\left(A B \cdot C q_{1}+O A\right)-B C\left(D E \cdot C q_{3}+E F\right) \\
f_{3}^{\prime}=-C D \cdot A B \cdot S q_{1} \cdot \dot{q}_{1}+B C \cdot D E \cdot C q_{3} \cdot \dot{q}_{3} \\
f_{3}^{\prime \prime}=-C D \cdot A B \cdot C q_{1} \cdot \dot{q}_{1}{ }^{2}-C D \cdot A B \cdot S q_{1} \cdot \ddot{q}_{1}-B C \cdot D E \cdot S q_{3} \cdot \dot{q}_{3}{ }^{2}+B C \cdot D E \cdot C q_{3} \cdot \ddot{q}_{3}
\end{gathered}
$$

Se establecen las condiciones iniciales para aplicar el Método de Newton Raphson y realizar la programación en Matlab:

- Altura mínima del mecanismo: $61.5 \mathrm{~mm}$

- Altura máxima del mecanismo: $306.5 \mathrm{~mm}$

- Ángulo inicial del eslabón $\mathrm{AB}, \mathrm{q} 1 \mathrm{o}=17.35^{\circ}$

- Ángulo inicial del eslabón BD, q2o = $167.57^{\circ}$

- Ángulo inicial del eslabón DE, q3o $=5.14^{\circ}$

Para determinar el valor de $\mathrm{q} 4 \mathrm{p}$ se toma en cuenta el avance del tornillo que es de $3 \mathrm{~mm} /$ revolución en un segundo. Entonces, se mide el desplazamiento de $3 \mathrm{~mm}$ en el tornillo para la altura inicial y final del mecanismo, una vuelta del tornillo:

Avance vertical

$27,68 \mathrm{~mm}-30,68 \mathrm{~mm}$

Avance horizontal

$95 \mathrm{~mm}-118,17 \mathrm{~mm}$

$$
\begin{gathered}
\uparrow v=\frac{(118,17-95) \mathrm{mm}}{1 \mathrm{~s}} \\
\uparrow v=23,17 \mathrm{~mm} / \mathrm{s} \\
v=0,02317 \mathrm{~m} / \mathrm{s}(q 4 p)
\end{gathered}
$$

La velocidad será constante por lo tanto la aceleración nula, q4pp = 0 . 
El análisis dinámico se lo realizó por el método de Potencias Virtuales, para lo cual se planteó las ecuaciones de cada parte:

- Eslabón AB:

$$
\underset{P_{A B}}{\longrightarrow} \cdot \frac{\partial \overrightarrow{V_{g 1}}}{\partial \dot{q}_{l}}+\overrightarrow{F_{l n 1}} \cdot \frac{\partial \overrightarrow{V_{g 1}}}{\partial \dot{q}_{l}}+\overrightarrow{T_{l n 1}} \cdot \frac{\partial \overrightarrow{\omega_{1}}}{\partial \dot{q}_{l}}
$$

- Eslabón BD:

$$
\overrightarrow{P_{B D}} \cdot \frac{\partial \overrightarrow{V_{g 2}}}{\partial \dot{q}_{\imath}}+\overrightarrow{F_{l n 2}} \cdot \frac{\partial \overrightarrow{V_{g 2}}}{\partial \dot{q}_{\iota}}+\overrightarrow{T_{l n 2}} \cdot \frac{\partial \overrightarrow{\omega_{2}}}{\partial \dot{q}_{\iota}}+\overrightarrow{F_{T}} \cdot \frac{\partial \overrightarrow{V_{2}}}{\partial \dot{q}_{\imath}}
$$

- Eslabón DE:

$$
\underset{P_{D E}}{\longrightarrow} \cdot \frac{\partial \overrightarrow{V_{g 3}}}{\partial \dot{q}_{l}}+\overrightarrow{F_{l n 3}} \cdot \frac{\partial \overrightarrow{V_{g 3}}}{\partial \dot{q}_{l}}+\overrightarrow{T_{l n 3}} \cdot \frac{\partial \overrightarrow{\omega_{3}}}{\partial \dot{q}_{l}}
$$

- Eslabón EF:

$$
\overrightarrow{P_{E F}} \cdot \frac{\partial \overrightarrow{V_{g 4}}}{\partial \dot{q}_{\iota}}+\overrightarrow{F_{l n 4}} \cdot \frac{\partial \overrightarrow{V_{g 4}}}{\partial \dot{q}_{\iota}}+\overrightarrow{F_{C}} \cdot \frac{\partial \overrightarrow{V_{4}}}{\partial \dot{q}_{\iota}}
$$

El análisis en Matlab se realizó en tres partes:

- Newton-Raphson

- Ecuaciones por el método de Potencias Virtuales

- Análisis Cinemático y Dinámico, obtención de las curvas.

\section{ANÁLISIS DE RESISTENCIA}

\section{- ESLABONES}

Para la obtención de tensiones de cada elemento en el software, se determinará mediante el estudio estático en SolidWorks enumerando fuerzas de vigas:

Debido a que la fuerza va a ser soportada por el mecanismo que está compuesto por 4 submecanismos iguales y simétricos, la fuerza se divide entre 4: 
Fuerza vertical a soportar:

$500 \mathrm{~kg} / 4=125 \mathrm{~kg}=1226,25 \mathrm{~N}$

Asimismo, como el tornillo va a estar sujeto entre 2 mecanismos simétricos, la fuerza se divide entre 2:

Fuerza horizontal del tornillo:

$5400 \mathrm{~N} / 2=2700 \mathrm{~N}$

Se determinan las fuerzas de tensión y compresión utilizando software de diseño asistido por computador, identificado el esquema de la figura 5:

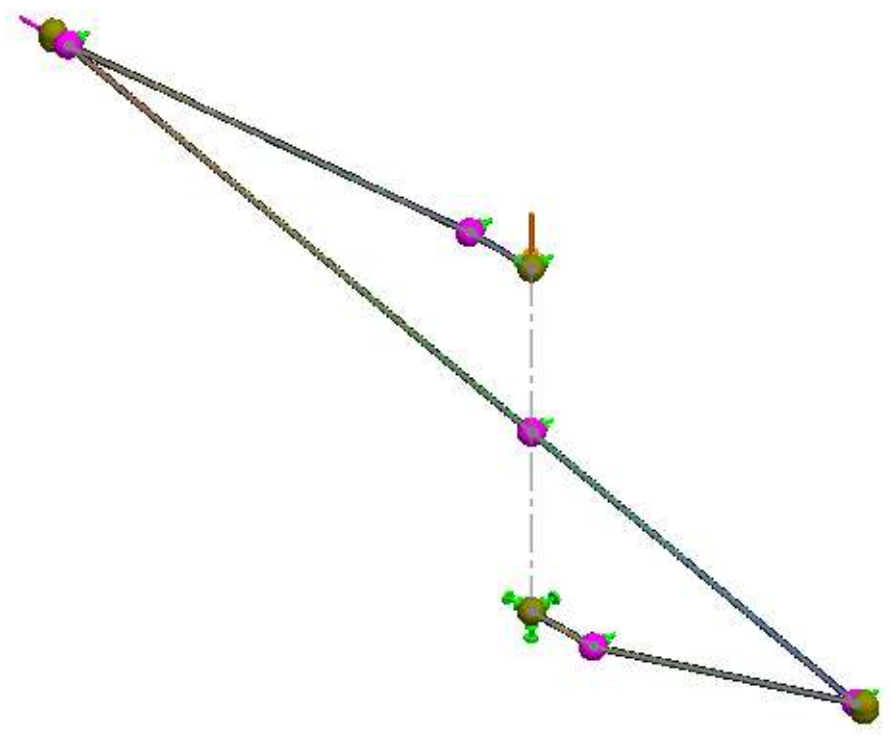

Fig. 5 Esquema de fuerzas de tensión y compresión

Del esquema de la figura 6 se obtienen las fuerzas de tensión y compresión de los elementos del mecanismo con los resultados mostrados en la figura 6: 


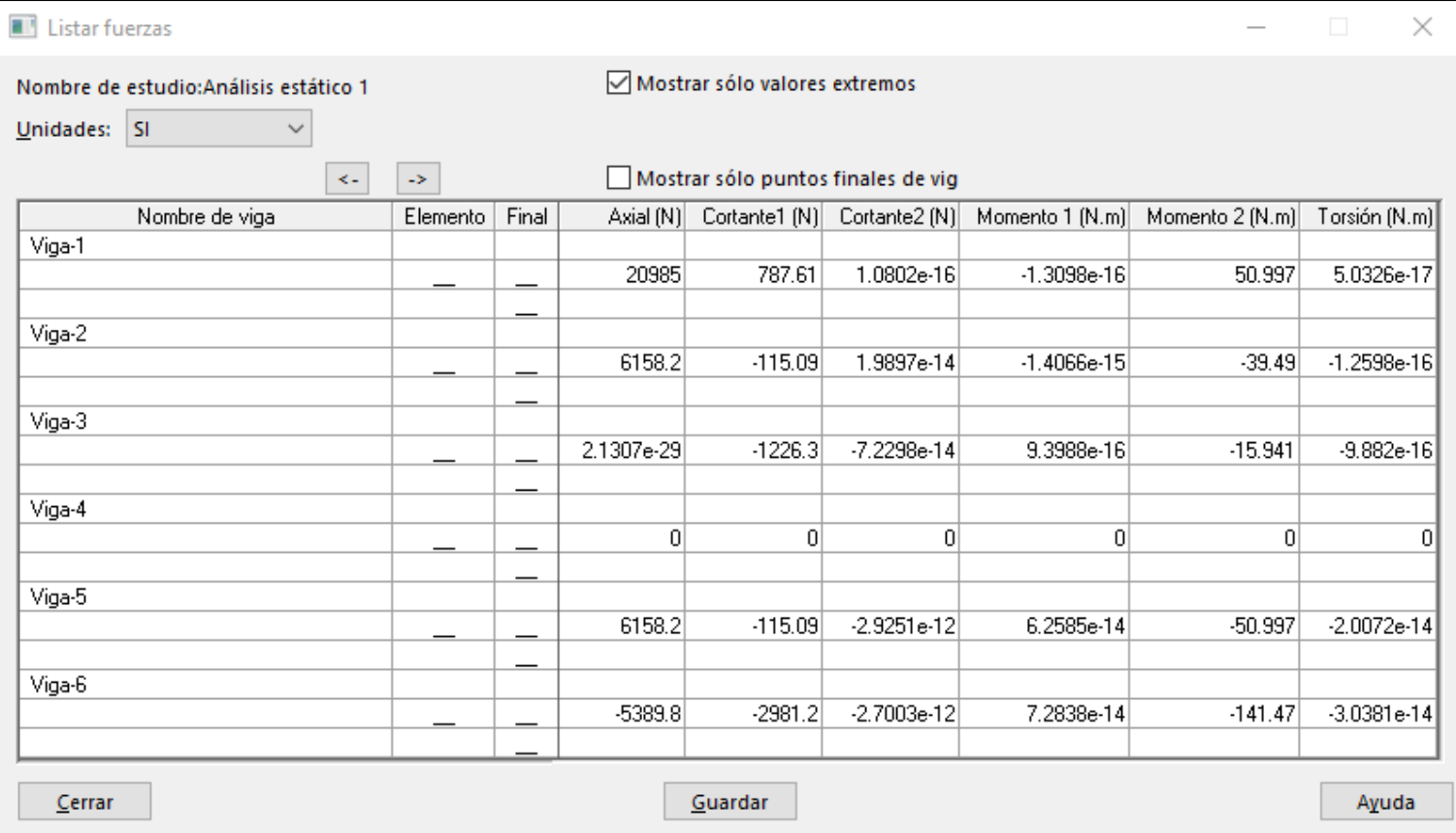

Fig. 6 Fuerzas de tensión y compresión en los eslabones

Eslabón AB: 5389,8 N a compresión

Eslabón BD: 6158,2 N a tensión

Eslabón DE: 20985 N a tensión

Para el análisis de resistencia, se determinaron las fuerzas de tensión y compresión en cada elemento, obteniendo los siguientes resultados comprobados con el factor de seguridad:

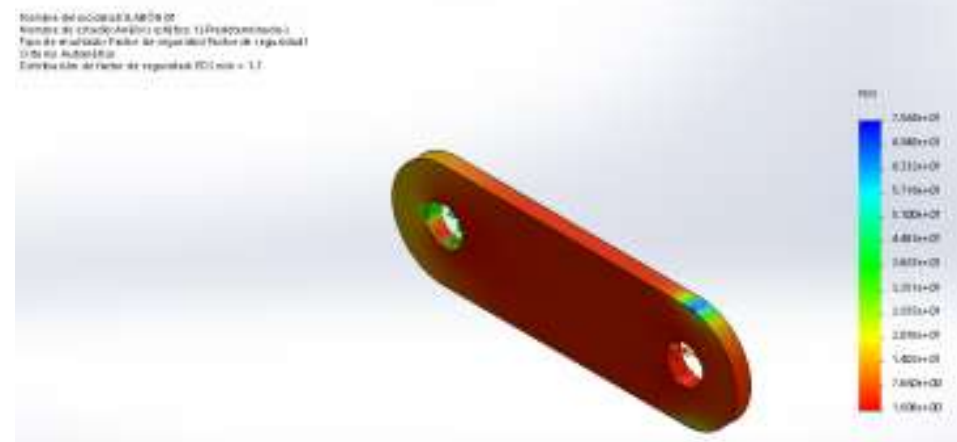

Fig. 7 Análisis de resistencia eslabón $\mathrm{AB}$ 


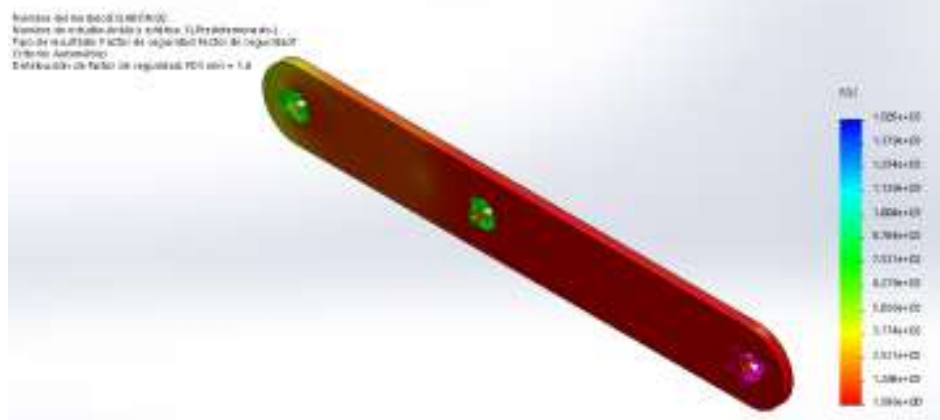

Fig. 8 Análisis de resistencia eslabón BD

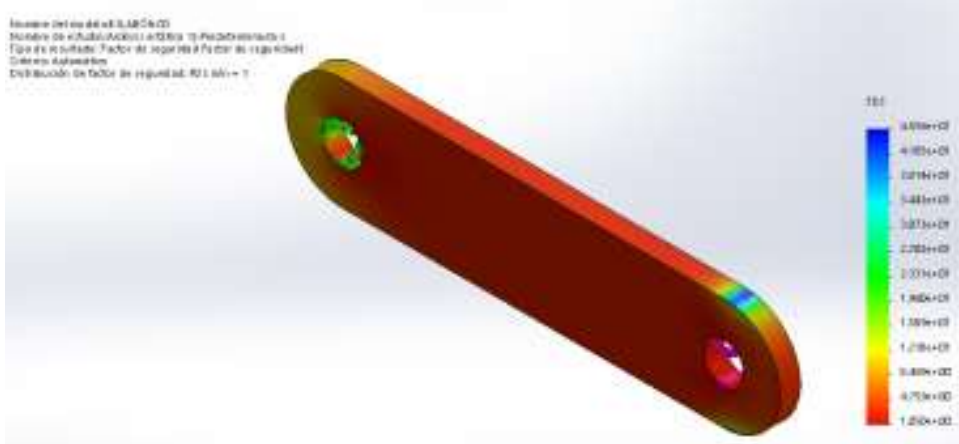

Fig. 9 Análisis de resistencia eslabón DE

\section{- MECANISMO}

Se ubica la carga de $5000 \mathrm{~N}$ en la parte superior del mecanismo:

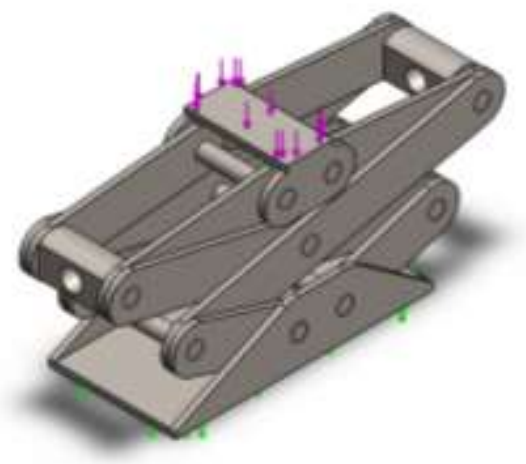

Figura 10: Ubicación de la carga en el mecanismo en SolidWorks 


\section{- MALLADO}

En las primeras etapas del análisis de diseño donde los resultados aproximados pueden resultar suficientes, puede especificar un tamaño de elemento mayor para una solución más rápida. Para obtener una solución más precisa, es posible que sea necesario utilizar un tamaño de elemento más pequeño.

Entonces se establece una configuración de malla intermedia:

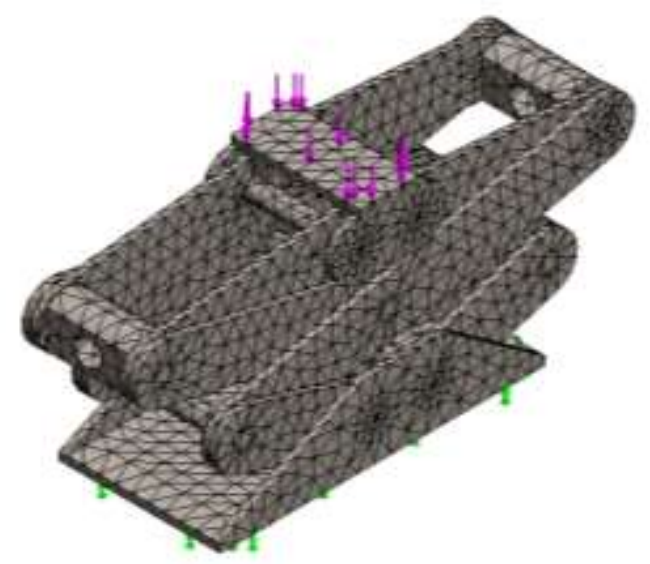

Figura 11: Mallado del mecanismo en SolidWorks

Y los resultados del diseño estático, no sobrepasa el límite estático:

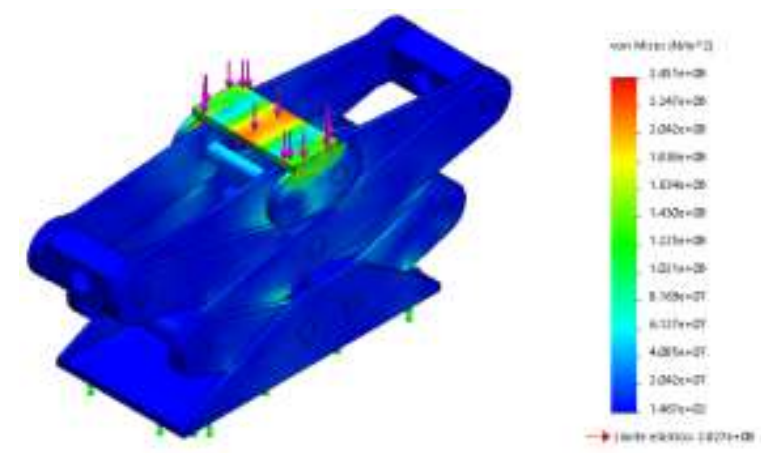

Figura 12: Límite elástico del mecanismo en SolidWorks Fuente: Autores

Factor de seguridad de 1.2: 


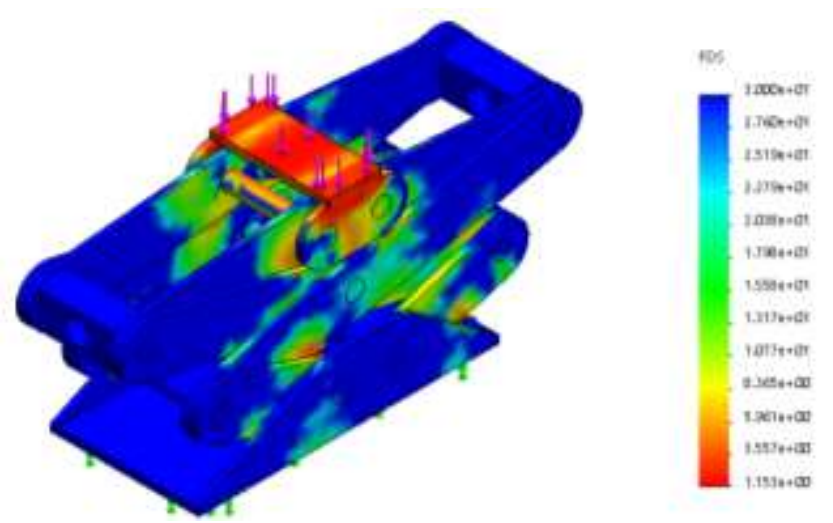

Figura 13: Factor de seguridad del mecanismo en SolidWorks

Para la comprobación a fatiga, se realiza con 10000 ciclos de carga y el resultado es que se obtendrá un porcentaje de daño del $68,3 \%$ :

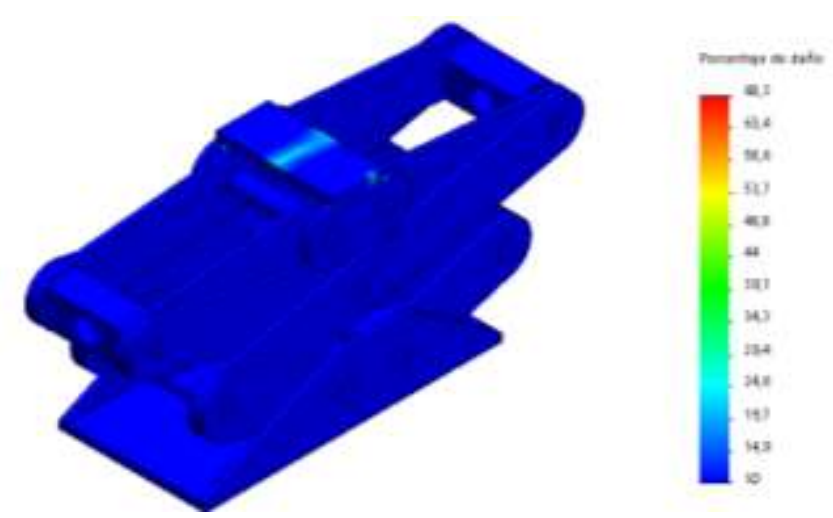

Figura 14: Porcentaje de dado por ensayo a fatiga del mecanismo en SolidWorks

Y la vida mínima que puede tener el mecanismo es de 146470 ciclos de carga en el punto indicado en la figura:

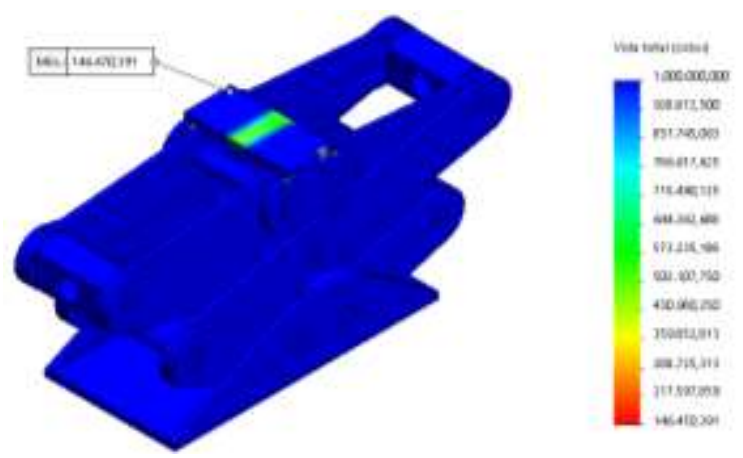

Figura 15: Vida útil mínima del mecanismo 


\section{Resultado y Discusión}

Como resultado del análisis cinemático se obtuvieron las ecuaciones de movimiento, velocidades y aceleraciones, teniendo como motriz a la altura del mecanismo, el mismo que está en función de la fuerza que se aplica en el tornillo para generar el movimiento.
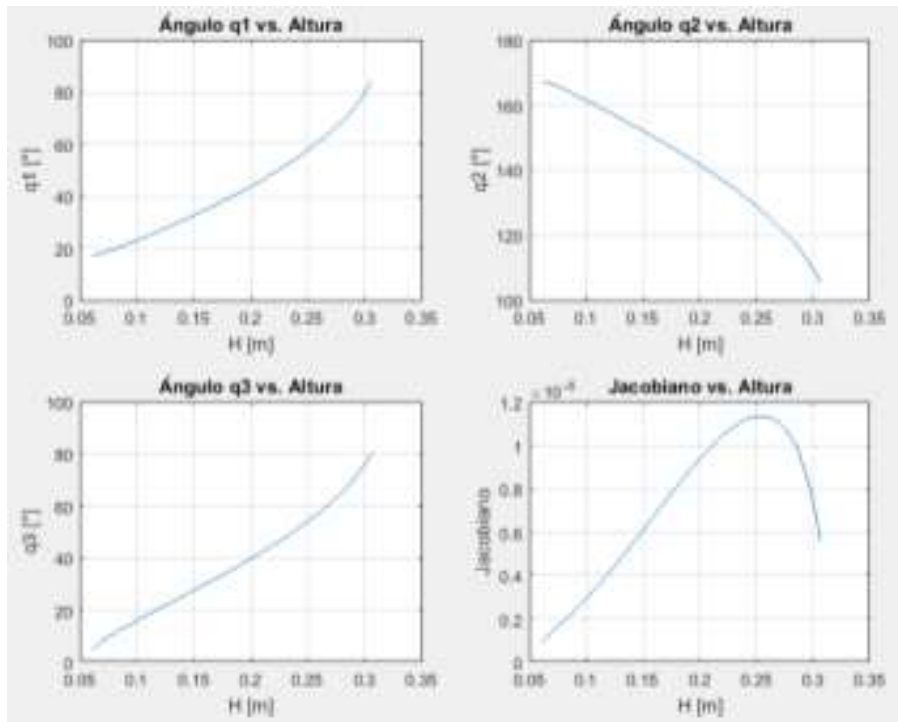

Fig. 16 Ángulos de los eslabones vs. Altura
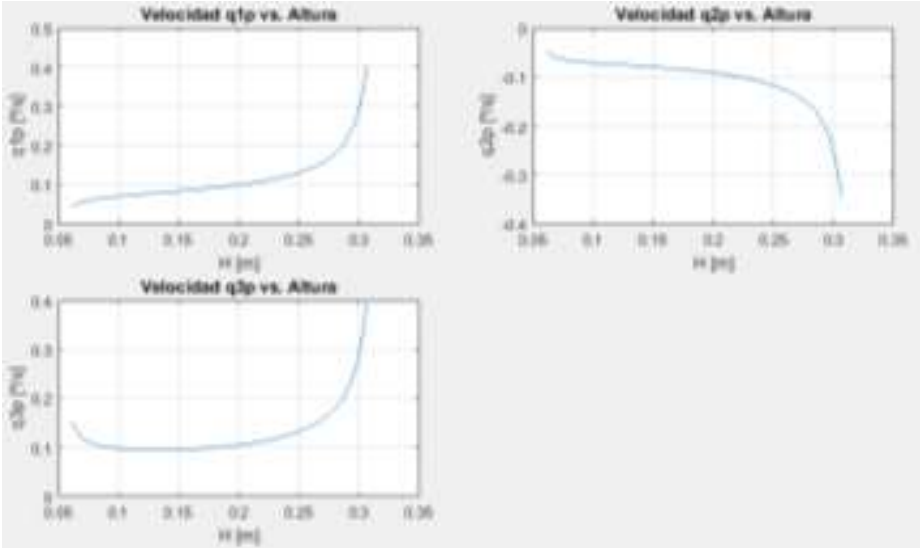

Fig. 17 Velocidades de los eslabones vs. Altura 


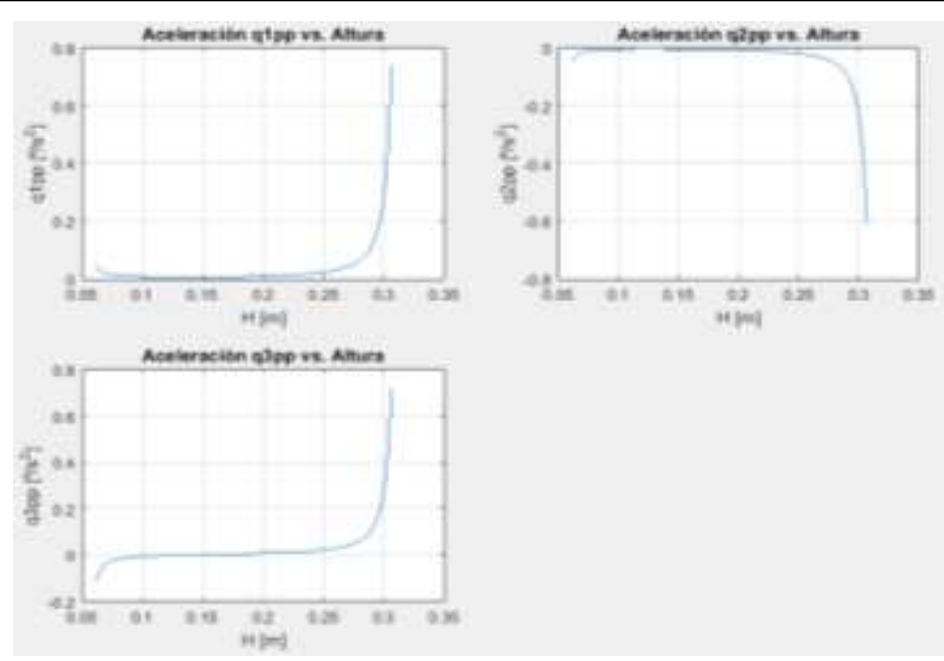

Fig. 18 Aceleraciones de los eslabones vs. Altura

En la figura 16 se observa los ángulos de los eslabones en función de la altura. Se puede observar que el ángulo del eslabón AB (q1) aumenta a medida que la altura sube, lo cual es lo esperado, pues es el primer eslabón que forma en ángulo q1 con el eslabón fijo horizontal OA. El ángulo q2 es el que se forma en el eslabón BD por lo que este inicial como obtuso y a medida que la altura aumente este disminuye, lo cual se puede observar en la gráfica respectiva. Finalmente, el eslabón DE que forma el ángulo q3 tiene variaciones similares a las de q1, lo cual se evidencia en la gráfica.

En la figura 17, se observan las curvas de la velocidad de los eslabones en función de la altura motriz. Se puede notar que las velocidades de los eslabones AB y DE que inician con un ángulo agudo (respecto a la horizontal), va aumentando a medida que la altura sube; mientras que el eslabón BD que inicia con un ángulo obtuso va disminuyendo a medida que la altura aumenta. Lo cual es geométricamente correcto.

En la figura 18 se observan las curvas de la aceleración de los eslabones en función de la altura motriz. Donde se evidencia que las aceleraciones de los eslabones se mantienen alrededor del valor 0 durante casi todo el movimiento, lo cual es esperado, ya que se planteó que la velocidad será constante.

Al final, el resultado obtenido más importante es el de la fuerza del tornillo en función de la altura de elevación del mecanismo según la Fig. 19. Donde se observa que la Fuerza requerida, a aplicarse en el tornillo, es menor a medida que la altura aumenta. Este parámetro sirve como base del análisis de resistencia del mecanismo. 


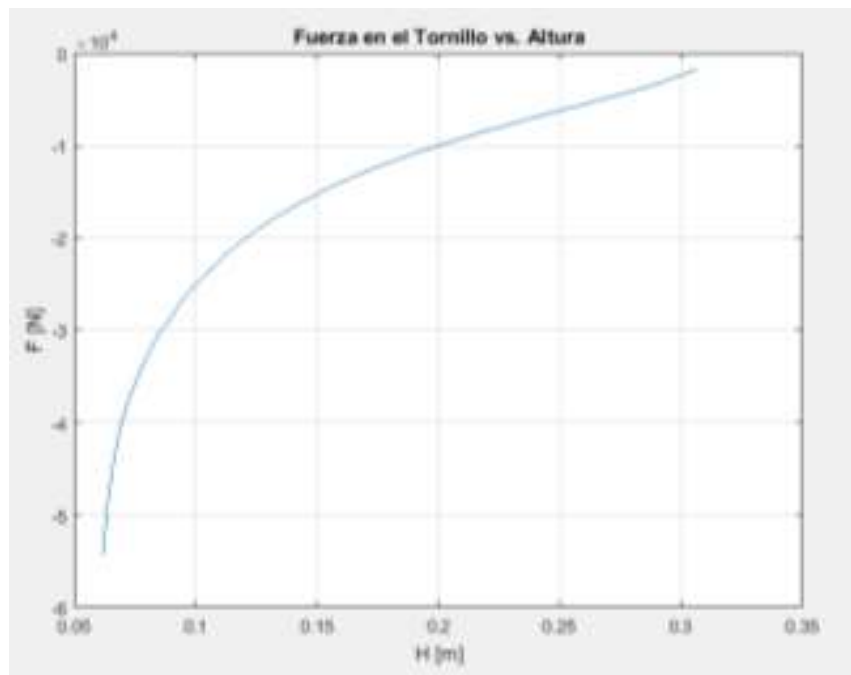

Fig. 19 Gráfica Fuerza en el Tornillo vs. Altura

Según las figuras 7, 8 y 9, se determina que el factor de seguridad obtenido en el diseño a resistencia mediante software de elementos finitos presente en los eslabones se encuentra comprendido entre 1 y 1.7 por lo que se establece que el mecanismo y material están optimizados al máximo.

En la figura 13 se identifica el menor factor de seguridad de 1,2 ubicado en la zona superior del soporte del mecanismo, en donde se puede realizar un reforzamiento o aumento de espesor para evitar que el elemento falle por cualquier sobrecarga. En el análisis de fatiga se determina una excelente vida útil del mecanismo, con los resultados de 10000 ciclos de carga, se obtiene un porcentaje de daño del 68,3\% y la vida mínima de por lo menos 146000 ciclos de carga, lo que quiere decir que el mecanismo durará por mucho.

\section{Conclusiones.}

- En la curva obtenida en el análisis cinemático, fuerza vs altura, se puede hallar el valor máximo en función de la altura, lo cual nos ayuda a encontrar la posición crítica del mecanismo, con esta información se pudo realizar un análisis de elemento finito en dicha posición.

- Los factores de seguridad están al límite lo que quiere decir que los elementos están bien optimizados para que desempeñen su función adecuadamente, en este caso eso es una ventaja, pues este mecanismo generalmente es portátil, al estar optimizado su peso es óptimo.

- Un inconveniente al aplicar este mecanismo sería la lentitud de accionamiento y también, de repliegue. Para ello se podría adaptar un motor, y de esta manera mejorar lo tiempos, formando un mecanismo más eficiente. 


\section{Referencias bibliográficas.}

Cuenca, M. J. (2000). Comentario de textos: los mecanismos referenciales. Retrieved from http://books.google.es/books/about/Comentario_de_textos.html?id=dBeAAAACAAJ\&pgis $=1$

De, D. E., Felker, L. G., \& Hill, I. A. (2005). Ecuaciones de Evans.

Forn, A. (2002). Envejecimiento En Materiales Compuestos Base. 859-866.

Industrial, D. (2011). Sistemas Oleohidráulicos. (2010), 62-69.

Ing Eduardo Torres Alpízar, C. (2004). Universidad de matanzas \&quot;camilo cienfuegos \&quot; : facultad de ingenierías química y mecanica. apuntes acerca del tratamiento térmico y la clasificación general de los aceros. Retrieved from http://monografias.umcc.cu/monos/2004/QuiMec/um04QM02.pdf

Luis Patricio Tierra Pérez Mg, I., Geovanny Guillermo Novillo Andrade Mg, I., \& Sócrates Miguel Aquino Arroba Mg, I. (2018). Mejora De La Calidad De Los Procesos En La Sección De Estructuras Con Metodología Seis Sigma. Caso Aplicado Industria Carrocera De Buses. 37-56.

Oliva, C. Z., Tamayo, E. T., Pazmiño, A. O., Pozo, E. R., Ordoñez Viñan, M. A., Aquino, S. M., \& Choto, L. S. (2019). Mathematical modeling of the coal activation process in rotary cylindrical kiln. 13, 15-20.

Ordoñez Viñan, M. A., Pozo-safla, E. R., Escobar-guachambala, M. A., \& Choto, L. S. (2018). Análisis cinemático de levas de baja velocidad, con leyes combinadas en base a métodos geométricos y matemáticos. 4, 185-205.

Snider, R. K. N. E. B. S. A. D. (2001). Ecuaciones diferenciales y problemas con valores en la frontera. Retrieved from https://books.google.com.ec/books?hl=es\&lr=\&id=b5H99FCGe_EC\&oi=fnd\&pg=PA $364 \& d q=$ ecuaciones+de+aceleración\&ots=DtHB58LMon\&sig=xUcfknw7N0w1ufgpy 9tJak9YZNI\# $\mathrm{v}=$ onepage \&q=ecuaciones de aceleración\&f=false

Uicker, J. J. (2016). Teoría de máquinas y mecanismos. (January).

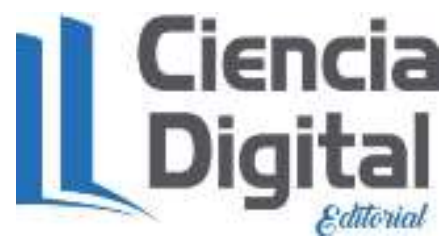


PARA CITAR EL ARTÍCULO INDEXADO.

Aquino Arroba, S., Tierra Pérez, L., Pozo Safla, E., \& Cazar Rivera, E. (2019). Modelación Matemática para la Dinámica de Mecanismos Hidráulicos Tipo Tijera. Ciencia Digital, 3(3.1), 74-90. https://doi.org/10.33262/cienciadigital.v3i3.1.677

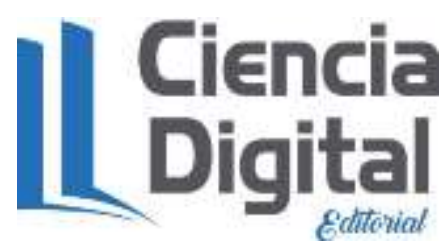

El artículo que se publica es de exclusiva responsabilidad de los autores y no necesariamente reflejan el pensamiento de la Revista Ciencia Digital.

El artículo queda en propiedad de la revista y, por tanto, su publicación parcial y/o total en otro medio tiene que ser autorizado por el director de la Revista Ciencia Digital.
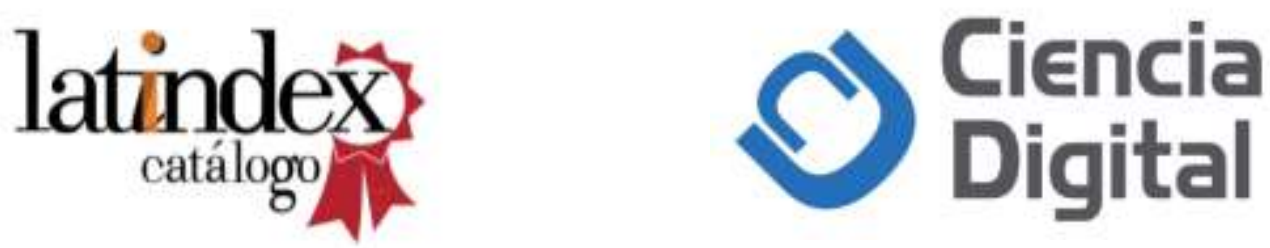